\title{
Quelques nouveaux phénomènes de rang 1 pour les groupes de difféomorphismes du cercle
}

\author{
Andrés Navas
}

Résumé. Nous démontrons un théorème de super-rigidité pour les actions de réseaux de rang supérieur par difféomorphismes du cercle.

Abstract. We prove a superrigidity theorem for actions of higher rank lattices by diffeomorphisms of the circle.

Mathematics Subject Classification (2000). 57M07, 58D05, 22F05, $37 \mathrm{E} 10$.

Mots-clés. Rigidité, cohomologie des groupes, difféomorphismes du cercle.

Keywords. Rigidity, group cohomology, circle diffeomorphisms.

\section{Introduction}

Le groupe des difféomorphismes du cercle partage quelques propriétés avec certains groupes de Lie simples de rang réel 1. L'un des phénomènes principaux qui permettent de justifier cette affirmation est donné par un théorème obtenu par l'auteur dans [27], lequel généralise dans plusieurs directions des résultats contenus dans [4], [5], [11], [16], [34], [39] et [40] (valables toutefois sous des hypothèses de régularité plus faibles). Rappelons qu'un groupe topologique localement compact possède la propriété ( $\mathrm{T}$ ) de Kazhdan si toute représentation affine (isométrique) de $\Gamma$ sur un espace de Hilbert admet un vecteur invariant.

Théorème ([27]). Soit $\Gamma$ un sous-groupe de $\operatorname{Diff}{ }_{+}^{1+\alpha}\left(\mathrm{S}^{1}\right)$, avec $\alpha>1 / 2$. Si $\Gamma$ possède la propriété (T) de Kazhdan, alors soit il est fini, soit il est topologiquement conjugué au groupe des rotations.

Une question qui se pose de manière naturelle est celle de savoir si les sous-groupes localement compacts de Diff ${ }_{+}^{1+\alpha}\left(\mathrm{S}^{1}\right), \alpha>1 / 2$, sont toujours (T)-moyennables (c'està-dire s'ils admettent des représentations affines géométriquement propres sur des 
espaces de Hilbert). L'une des difficultés de cette question est le fait que l'on connaît très peu de groupes qui ne sont pas (T)-moyennables et n'ont pas non plus la propriété (T). En effet, la seule obstruction qu'on connaît pour la (T)-moyennabilité est une forme faible de la propriété $(\mathrm{T})$, à savoir la propriété $(\mathrm{T})$ relative.

Rappelons que si $\Gamma$ est un groupe localement compact et $\Gamma_{0}$ est un sous-groupe de $\Gamma$, alors on dit que la paire $\left(\Gamma, \Gamma_{0}\right)$ possède la propriété $(\mathrm{T})$ relative si pour toute représentation affine de $\Gamma$ sur un espace de Hilbert, il existe un vecteur invariant par $\Gamma_{0}$. Un exemple non trivial d'une paire satisfaisant la propriété $(T)$ relative est $\left(\mathbb{Z}^{2} \rtimes \operatorname{SL}(2, \mathbb{Z}), \mathbb{Z}^{2}\right)$. On trouve d'autres exemples ainsi que des reférences sur le sujet dans [9] et [18]. Notons que pour tous les exemples connus, si aucun des groupes $\Gamma$ ou $\Gamma_{0}$ n'a déjà la propriété $(\mathrm{T})$, alors $\Gamma_{0}$ contient un sous-groupe cocompact $\Gamma_{0}^{\prime}$ qui est distingué dans un sous-groupe $\Gamma^{\prime}$ de $\Gamma$ de sorte que $\left(\Gamma^{\prime}, \Gamma_{0}^{\prime}\right)$ vérifie encore la propriété (T) relative (voir cependant [10]). Sous une telle hypothèse, nous démontrons le résultat suivant, lequel peut être considéré comme une petite généralisation du théorème énoncé précédemment.

Théorème A. Soit $\Gamma$ un sous-groupe de $\operatorname{Diff}_{+}^{1+\alpha}\left(\mathrm{S}^{1}\right)$, avec $\alpha>1 / 2$. Supposons que $\Gamma$ possède un sous-groupe distingué $\Gamma_{0}$ tel que la paire $\left(\Gamma, \Gamma_{0}\right)$ satisfait la propriété (T) relative. Alors soit $\Gamma$ est topologiquement conjugué à un groupe de rotations, soit $\Gamma_{0}$ est fini.

La démonstration de ce théorème est inspirée de [27]. L'amélioration technique essentielle consiste en une preuve courte et conceptuelle d'une proposition énoncée (et non démontrée) dans [27], suivant laquelle les sous-groupes de $\operatorname{Diff}_{+}^{1+\alpha}\left(S^{1}\right)$, $\alpha>1 / 2$, sur lesquels le « cocycle de Liouville $\gg$ est cohomologiquement trivial sont topologiquement conjugués à des sous-groupes de PSL $(2, \mathbb{R})$.

Rappelons que les réseaux de groupes de Lie simples de rang supérieur satisfont la propriété (T). Le théorème dans [27] donne donc en particulier une nouvelle démonstration (en classe $\mathrm{C}^{1+\alpha}, \alpha>1 / 2$ ) de l'un des résultats obtenus par É. Ghys dans [16] et indépendamment par M. Burger et N. Monod dans [4], à savoir pour toute représentation $\phi$ d'un réseau $\Gamma$ d'un groupe de Lie simple de rang réel supérieur ou égal à 2 dans le groupe des difféomorphismes directs et de classe $C^{1}$ du cercle, l'image $\phi(\Gamma)$ est finie. Néanmoins, dans [16], É. Ghys obtient également la classification des actions de réseaux irréductibles de groupes de Lie semi-simples de rang supérieur par difféomorphismes directs et de classe $\mathrm{C}^{1}$ du cercle (voir aussi [5] et le $\$ 14$ de [23], où $\mathrm{M}$. Burger et $\mathrm{N}$. Monod obtiennent des résultats analogues grâce à leur étude de la cohomologie bornée). Signalons en passant que dans [4], [5] et [16], on trouve des résultats partiels pour des actions par homéomorphismes du cercle.

Théorème ([16]). Soit $G$ un groupe de Lie semi-simple connexe de rang réel supérieur ou égal à 2 et $\Gamma$ un réseau irréductible de $G$. Soit $\phi$ un homomorphisme de $\Gamma$ vers le groupe des difféomorphismes de classe $\mathrm{C}^{1}$ du cercle respectant l'orientation. Alors, 
ou bien $\phi$ a une image finie cyclique, ou bien $\phi$ est semiconjugué à un revêtement fini d'un homomorphisme obtenu en faisant suivre :

- le plongement de $\Gamma$ dans $G$,

- une surjection de $G$ sur $\operatorname{PSL}(2, \mathbb{R})$,

- l'action projective de $\operatorname{PSL}(2, \mathbb{R})$ sur le cercle (identifié à la droite projective réelle).

Pour parvenir à la preuve de ce résultat, É. Ghys examine d'abord le cas des réseaux de groupes de Lie semi-simples «classiques $\gg(\operatorname{SL}(n, \mathbb{R}), \operatorname{Sp}(2 r, \mathbb{R}), \mathrm{SO}(2, q)$, $\operatorname{SU}(2, q)$ et $\operatorname{PSL}(2, \mathbb{R}) \times \operatorname{PSL}(2, \mathbb{R}))$, et puis en utilisant quelques aspects de la théorie de classification des groupes de Lie semi-simples, il aboutit au résultat énoncé. Remarquons que les quatre premiers cas correspondent à des groupes de Lie simples de rang réel supérieur ou égal à 2 (les réseaux correspondants vérifient donc la propriété (T) de Kazhdan). Le dernier cas est dynamiquement plus intéressant. Dans cette situation, É. Ghys démontre que tout homomorphisme $\phi: \Gamma \rightarrow \operatorname{Diff}_{+}^{1}\left(\mathrm{~S}^{1}\right)$ transite, modulo une semiconjugaison et un revêtement fini, par la projection de $\Gamma$ sur l'un des facteurs, et puis par l'action projective de ce facteur sur le cercle.

Pour généraliser le théorème de Ghys ci-dessus, on est confronté au problème de définir la notion de rang réel pour un groupe quelconque. Bien que plusieurs tentatives ont été déjà faites dans cette direction (voir par exemple [2]), nous suivrons plutôt une idée très simple qui a été introduite par Y. Shalom dans [35]. Le point essentiel de son approche consiste à tirer partie de la commutativité des facteurs d'un groupe, vue comme une hypothèse de rang supérieur. Ainsi, le «cadre général» que nous considérerons - et qui est aussi celui de [35] - est le suivant :

(a) $G=G_{1} \times \cdots \times G_{k}$ est un groupe topologique compactement engendré, avec $k \geq 2$, et $\Gamma$ est un réseau de type fini et uniforme (c'est-à-dire un sous-groupe discret et cocompact) de $G$;

(b) les projections de $\Gamma$ sur chaque facteur $G_{i}$ sont denses (nous désignérons $\operatorname{par} \mathrm{pr}_{i}$ la projection de $G \operatorname{sur} G_{i}$ );

(c) dans le cas où chaque $G_{i}$ est un groupe algébrique linéaire sur un corps local, on acceptera aussi la possibilité que $\Gamma$ soit un réseau non cocompact dans $G$.

Remarquons que dans le cas (c) ci-dessus, le réseau $\Gamma$ est automatiquement de type fini. Ceci découle de certains résultats de D. Kazhdan et G. Margulis. D'autre part, la condition (b) est une condition d'irréductibilité comme celle des réseaux considérés dans le théorème de Ghys.

Dans l'introduction de [35] on peut trouver des motivations ainsi que des références concernant le cadre général que nous considérons. Signalons en tout cas que des exemples de réseaux «non linéaires» vérifiant les propriétés (a) et (b) ont été construits dans [3], [6] et [33]. Pour ces réseaux, ainsi que pour les réseaux irréductibles «linéaires», nous obtenons le résultat de super-rigidité suivant. 
Théorème B. Dans le cadre précédent, supposons que $\phi: \Gamma \rightarrow \operatorname{Diff}_{+}^{1+\alpha}\left(\mathrm{S}^{1}\right)$ soit un homomorphisme tel que $\alpha>1 / 2$ et tel que $\phi(\Gamma)$ ne préserve aucune mesure de probabilité sur le cercle. Alors soit $\phi(\Gamma)$ est topologiquement conjugué à un sous-groupe de $\operatorname{PSL}(2, \mathbb{R})$, soit $\phi$ est semiconjugué à un revêtement fini d'un homomorphisme obtenu en faisant suivre :

- le plongement de $\Gamma$ dans $G$,

- la projection de $G$ sur l'un des $G_{i}$,

- une action $\Phi$ de $G_{i}$ par homéomorphismes du cercle.

L'hypothèse suivant laquelle $\phi(\Gamma)$ ne fixe aucune mesure de probabilité du cercle peut être supprimée, pourvu que l'on suppose que le premier espace de cohomologie à valeurs réels de tout sous-groupe distingué et d'indice fini de $\Gamma$ soit trivial. Remarquons que d'après [35], cette hypothèse est vérifiée lorsque $\mathrm{H}^{1}(G, \mathbb{R})$ est trivial (c'est le cas par exemple si les $G_{i}$ sont des groupes linéaires algébriques semi-simples sur des corps locaux).

Corollaire. Soit $\Gamma$ un réseau vérifiant les hypothèses du cadre général et $\phi: \Gamma \rightarrow$ $\operatorname{Diff}_{+}^{1+\alpha}\left(\mathrm{S}^{1}\right)$ un homomorphisme, avec $\alpha>1 / 2$. Si $\mathrm{H}^{1}\left(\Gamma_{0}, \mathbb{R}\right)=\{0\}$ pour tout sous-groupe $\Gamma_{0}$ d'indice fini et distingué dans $\Gamma$, alors la conclusion du théorème $B$ ci-dessus est encore satisfaite.

D'après ce qui précède, pour comprendre les actions de réseaux irréductibles de rang supérieur par difféomorphismes du cercle, il suffit de connaître les actions de groupes topologiques compactement engendrés. Or, ces groupes étant localement compacts, une application simple du théorème de Montgomery et Zippin montre que $\Phi\left(G_{i}\right)$ a une structure de groupe de Lie (réel). D'autre part, on connaît parfaitement la classification des actions fidèles de groupes de Lie connexes par homéomorphismes directs du cercle : ces actions sont induites par celles du groupe des rotations $\operatorname{SO}(2, \mathbb{R})$, du groupe des translations $(\mathbb{R},+)$, du groupe affine $\operatorname{Aff}_{+}(\mathbb{R},+)$, du groupe projectif et ses revêtements finis $\operatorname{PSL}_{k}(2, \mathbb{R}), k \geq 1$, ainsi que de son revêtement universel $\widetilde{P S L}(2, \mathbb{R})$. En nous appuyant sur cette classification, nous pouvons donner une version plus précise du théorème $\mathrm{B}$ sous l'une quelconque des hypothèses suivantes :

(i) le noyau de $\phi$ est fini et les orbites de $\phi(\Gamma)$ sont denses,

(ii) le noyau de $\phi$ est fini et $\phi$ est à valeurs dans le groupe $\operatorname{Diff}_{+}^{\omega}\left(\mathrm{S}^{1}\right)$ des difféomorphismes directs et analytiques réels du cercle,

(iii) les sous-groupes distingués de $\Gamma$ sont soit finis soit d'indice fini (c'est-à-dire que $\Gamma$ satisfait le théorème des sous-groupes distingués de Margulis).

Théorème C. Supposons que les hypothèses du corollaire soient vérifiées, que chaque groupe $G_{i}$ soit non discret, et qu'au moins l'une des hypothèses (i), (ii) ou (iii) 
Vol. 80 (2005) Phénomènes de rang 1 pour les groupes de difféomorphismes du cercle 359

ci-dessus soit satisfaite. Si l'image $\phi(\Gamma)$ n'est pas finie alors, à semiconjugaison topologique et à revêtement fini près, $\phi(\Gamma)$ est un sous-groupe non métabélien de $\operatorname{PSL}(2, \mathbb{R})$.

Les hypothèses (i), (ii) ou (iii) sont faites de sorte à éviter le cas éventuel d'un groupe de Lie avec une infinité de composantes connexes dont l'action sur le cercle transite, à semiconjugaison topologique près, à travers du quotient par rapport à la composante connexe de l'identité. Signalons que l'hypothèse (iii) est satisfaite par les réseaux de groupes algébriques, ainsi que par ceux qui sont construits dans [6] (voir [1] pour une version générale de ce fait).

Un corollaire intéressant de ce qui précède est le fait que le groupe $\mathrm{G}$ de Thompson n'est pas un réseau vérifiant les hypothèses du théorème $C$. Remarquons toutefois que le fait que $\mathrm{G}$ n'est pas un réseau dans un groupe algébrique sur un corps local découle du fait que G, étant un groupe simple, n'est pas résiduellement fini. De plus, sa dimension cohomologique est infinie (voir [17]).

La technique de démonstration des théorèmes $\mathrm{B}$ et $\mathrm{C}$ est fortement inspirée par les résultats obtenus par Y. Shalom dans [35], notamment le théorème de super-rigidité cohomologique. Remarquons par ailleurs qu'avec nos résultat et le théorème d'arithmeticité 0.5 de [35], il est possible de donner dans notre contexte une description précise (du point de vue algébrique) des réseaux qui agissent sur le cercle (avec image infinie). Ceci permet d'utiliser l'argument du $\$ 10$ de [16] pour obtenir des conjugaisons lisses dès que les actions sont suffisamment différentiables (on obtient en particulier de véritables conjugaisons topologiques pour des morphismes à valeurs dans $\left.\operatorname{Diff}_{+}^{2}\left(S^{1}\right)\right)$.

Signalons finalement que Y. Neretin a introduit un groupe (à savoir, le groupe des sphéromorphismes, connu aussi sous le nom de groupe de Neretin) qui est un analogue combinatoire (ou $p$-adique) du groupe des difféomorphismes du cercle. En utilisant une technique introduite par D. Farley dans [12], nous avons établi dans [28] un résultat analogue à celui de [27] pour ce groupe, qui étend également le théorème classique d'Alperin et Watatani dans le cas d'un arbre simplicial homogène (voir [18]). Nous ignorons si l'on peut obtenir un analogue du théorème $B$ pour le groupe de Neretin. Un tel résultat serait une généralisation du théorème de super-rigidité pour des actions isométriques sur des arbres obtenu par Y. Shalom dans [35] (voir aussi [24]).

\section{Rappels cohomologiques}

2.1. Cohomologie continue. Soit $\theta$ une représentation unitaire d'un groupe topologique localement compact $\Gamma$ sur un espace de Hilbert $\mathscr{H}$. On dit que $c: \Gamma \rightarrow \mathcal{H}$ est un cocycle par rapport à $\theta$ si $c$ est une application continue et si la correspondance 
$g \mapsto \theta(g)+c(g)$ définit une représentation affine de $\Gamma$, ce qui revient à dire que pour tout $g, h \in \Gamma$ on a $c(g h)=c(g)+\theta(g) c(h)$. On dit qu'un cocycle $c$ est un cobord s'il existe $K \in \mathscr{H}$ qui est fixé par l'action affine induite, ce qui se traduit par le fait que $c(g)=K-\theta(g) K$ pour tout $g \in \Gamma$. Le premier espace de cohomologie (continue) $\mathrm{H}^{1}(\Gamma, \theta)$ est défini comme le quotient entre l'espace des cocycles et le sous-espace des cobords. Un groupe topologique localement compact $\Gamma$ possède la propriété $(\mathrm{T})$ de Kazhdan si pour toute représentation $\theta$ continue et unitaire de $\Gamma$, l'espace $\mathrm{H}^{1}(\Gamma, \theta)$ est trivial.

Comme nous l'avons déjà signalé dans l'introduction, le fait que Diff ${ }_{+}^{1+\alpha}\left(\mathrm{S}^{1}\right)$ ne contient pas de sous-groupe de Kazhdan non compact pour $\alpha>1 / 2$ est un résultat obtenu dans [27]. Le problème de savoir s'il existe des sous-groupes (non triviaux) de Homéo $_{+}(\mathbb{R})$ vérifiant la propriété $(\mathrm{T})$ est ouvert. Quant au cas des difféomorphismes de l'intervalle, rappelons que d'après le théorème de stabilité de Thurston, tout sous-groupe $\Gamma$ de Diff ${ }_{+}^{1}([0,1])$ de type fini et non réduit à l'identité admet un homomorphisme non trivial $\phi: \Gamma \rightarrow(\mathbb{R},+$ ) (voir [7] pour une preuve élémentaire de ce résultat ou [37] pour la démonstration originale). Puisque la propriété (T) passe aux quotients et puisque les groupes abéliens de type fini et non finis n'ont pas la propriété (T), ceci implique qu'aucun sous-groupe de type fini et non trivial de Diff ${ }_{+}^{1}([0,1])$ n'a la propriété $(\mathrm{T})$.

Une preuve simple de ce dernier fait apparaît dans [41]. En manière d'exemple, nous le redémontrerons sous une hypothèse de régularité supplémentaire. La preuve ci-dessus est inspirée de [27].

Exemple 2.1. Rappelons d'abord que $\operatorname{Diff}_{+}^{1+\alpha}([0,1])$ (resp. Diff $\left.{ }_{+}^{1+\alpha}\left(S^{1}\right)\right)$ désigne le groupe des difféomorphismes directs de l'intervalle (resp. du cercle) qui ont une dérivée Hölder continue d'exposant $\alpha>0$, avec un inverse satisfaisant la même propriété.

Soit $\Gamma$ un sous-groupe de $\operatorname{Diff}_{+}^{1+\alpha}([0,1])$, où $\alpha>0$. Quitte à considérer un quotient de $\Gamma$, on peut supposer qu'il n'admet pas de point (globalement) fixe sur ]0, 1[. Considérons la mesure de Radon $d \mu=d x / x$ sur ]0, 1 [, et notons $\theta$ la représentation régulière de $\Gamma$ dans $\mathscr{H}=\mathscr{L}_{\mathbb{R}}^{2}([0,1], d \mu)$, c'est-à-dire

$$
\theta\left(g^{-1}\right) K(x)=K(g(x))\left[\frac{d g}{d \mu}(x)\right]^{1 / 2} .
$$

Pour chaque $g \in \Gamma$ considérons la fonction

$$
c\left(g^{-1}\right)=1-\left[\frac{d g}{d \mu}(x)\right]^{1 / 2} .
$$

La relation de cocycle $c(g h)=c(g)+\theta(g) c(h)$ est satisfaite, car $c$ est le cobord formel de la fonction constante égale à 1 (qui n'appartient pas à $\mathscr{H}$ ). Nous affirmons d'autre 
Vol. 80 (2005) Phénomènes de rang 1 pour les groupes de difféomorphismes du cercle 361

part que $c(g)$ appartient à $\mathscr{H}$. En effet, si $g \in \Gamma$ et $x \in] 0,1\left[\right.$ alors $g(x)=x g^{\prime}(y)$ pour certain $y \in] 0, x[$, et donc

$$
\frac{x g^{\prime}(x)}{g(x)}=\frac{x g^{\prime}(x)}{x g^{\prime}(y)}=\frac{g^{\prime}(x)}{g^{\prime}(y)}
$$

En notant $\bar{C}=\inf _{x \in[0,1]} g^{\prime}(x)$, du fait que $\left|g^{\prime}(x)-g^{\prime}(y)\right| \leq C|x-y|^{\alpha} \leq C x^{\alpha}$ on obtient

$$
\begin{aligned}
\left|\left[\frac{1}{x}\right]^{1 / 2}-\left[\frac{g^{\prime}(x)}{g(x)}\right]^{1 / 2}\right| & =\frac{1}{x^{1 / 2}}\left|\left[\frac{g^{\prime}(x)}{g^{\prime}(y)}\right]^{1 / 2}-1\right| \\
& =\frac{1}{x^{1 / 2}} \cdot \frac{\left|g^{\prime}(x)-g^{\prime}(y)\right|}{g^{\prime}(y)^{1 / 2}\left(g^{\prime}(x)^{1 / 2}+g^{\prime}(y)^{1 / 2}\right)} \\
& \leq \frac{C}{2 \bar{C}} \cdot \frac{x^{\alpha}}{x^{1 / 2}},
\end{aligned}
$$

et cette dernière fonction appartient à $\mathcal{L}_{\mathbb{R}}^{2}([0,1], d x)$ dès que $\alpha>0$. Puisque

$$
\left\|c\left(g^{-1}\right)\right\|_{\mathcal{H}}^{2}=\int_{0}^{1}\left|\left[\frac{1}{x}\right]^{1 / 2}-\left[\frac{g^{\prime}(x)}{g(x)}\right]^{1 / 2}\right|^{2} d x,
$$

ceci montre l'affirmation.

Si $\Gamma$ possède la propriété $(\mathrm{T})$ alors il existe une fonction $K \in \mathscr{H}$ telle que $c(g)=$ $K-\theta(g) K$. En reprenant les définitions on vérifie que la mesure $v$ sur ]0, 1[ dont la fonction densité (par rapport à $d \mu$ ) est le carré de

$$
x \mapsto 1-K(x)
$$

est invariante par $\Gamma$. Cette mesure de Radon $v$ a une masse infinie sur ]0, 1[. D'autre part, on a $v(] \varepsilon, 1[)<\infty$ pour tout $\varepsilon>0$. Le fait que $\Gamma$ ne peut pas avoir la propriété (T) découle ainsi du lemme élémentaire suivant.

Lemme 2.2. Soit $v$ une mesure de Radon (non triviale) sur $] 0,1[$ telle que $v(] \varepsilon, 1[)<$ $\infty$ pour tout $\varepsilon>0$. Si $\Gamma$ est un sous-groupe de Homéo $_{+}([0,1])$ qui préserve $v$, alors $\Gamma$ admet des points (globalement) fixes sur $] 0,1[$.

Preuve. Fixons deux points $a$ et $b$ dans $] 0,1[$ tels que $a<b$ et $v([a, b[)>0$. Supposons que $\Gamma$ n'ait pas de point globalement fixe sur ]0, 1[ et désignons par $c \in[a, 1]$ le supremum de l'orbite de $a$ par $\Gamma$. Le point $c$ est globalement fixé, et donc $c=1$. Ceci entraîne l'existence d'un élément $g \in \Gamma$ tel que $g(a)>b$. On obtient ainsi $v([b, 1[)<v([a, b[)+v([b, 1[)=v([a, 1[)=v([g(a), 1[) \leq v([b, 1[)$, ce qui est absurde. 
Remarquons par ailleurs que si un sous-groupe de Homéo $o_{+}([0,1])$ préserve une mesure de Radon (non triviale) sur ]0, 1[, alors la fonction nombre de translation par rapport à $v$ fournit un homomorphisme de $\Gamma$ sur $(\mathbb{R},+)$, et cet homomorphisme est non trivial si et seulement si $\Gamma$ n'admet pas de point globalement fixe à l'intérieur de l'intervalle (voir [31]).

2.2. Cohomologie réduite. Étant donnée une représentation unitaire $\theta$ d'un groupe topologique localement compact $\Gamma$ sur un espace de Hilbert $\mathscr{H}$, on considère la topologie sur l'espace des cocycles suivant laquelle une suite $c_{n}$ de cocycles converge vers $c$ si et seulement si pour tout sous-ensemble compact $\mathrm{C}$ de $\Gamma$, la valeur de $\sup _{g \in \mathrm{C}}\left\|c_{n}(g)-c(g)\right\|$ converge vers zéro lorsque $n$ tend vers l'infini. Le premier espace de cohomologie réduite $\overline{\mathrm{H}}^{1}(\Gamma, \theta)$ est alors défini comme étant le quotient entre l'espace des cocycles et la fermeture du sous-espace des cobords. Signalons que dans [35], Y. Shalom a démontré que la propriété (T) peut être testée en cohomologie réduite pour les groupes compactement engendrés. Plus précisément, un groupe topologique compactement engendré $\Gamma$ possède la propriété $(\mathrm{T})$ de Kazhdan si et seulement si pour toute représentation $\theta$ continue et unitaire de $\Gamma$, l'espace $\overline{\mathrm{H}}^{1}(\Gamma, \theta)$ est trivial (voir aussi [22]).

Définition 2.3. La représentation unitaire $\theta$ possède presque des vecteurs invariants s'il existe une suite de vecteurs unitaires $K_{n} \in \mathscr{H}$ telle que pour tout sous-ensemble compact $\mathrm{C}$ de $\Gamma$, la valeur de $\sup _{g \in \mathrm{C}}\left\|K_{n}-\theta(g) K_{n}\right\|$ tend vers zéro lorsque $n$ tend vers l'infini.

Le lemme élémentaire suivant, dû à P. Delorme (voir [18]), s'avère fondamental pour étudier la cohomologie réduite. Nous en rappelons la preuve afin que le texte soit le plus autocontenu possible.

Lemme 2.4. Si $\theta$ n'a pas presque des vecteurs invariants alors l'injection de la cohomologie continue $\mathrm{H}^{1}(\Gamma, \theta)$ dans la cohomologie réduite $\overline{\mathrm{H}}^{1}(\Gamma, \theta)$ est un isomorphisme.

Preuve. Soit $c$ un cocycle associé à $\theta$ dont sa classe en cohomologie réduite est nulle. Supposons que $\theta$ n'ait pas presque des vecteurs invariants. Nous allons démontrer dans ce cas que $c$ est nul en cohomologie continue.

Soit $\Gamma^{1}$ une partie génératrice compacte de $\Gamma$. Par hypothèse, il existe une constante $\varepsilon>0$ telle que pour tout $K \in \mathscr{H}$,

$$
\sup _{h \in \Gamma^{1}}\|K-\theta(h) K\| \geq \varepsilon\|K\| .
$$

Puisque $c$ est nul en cohomologie réduite, il existe une suite $\left(K_{n}\right)$ dans $\mathscr{H}$ telle que pour tout $g \in \Gamma$ on a $c(g)=\lim _{n \rightarrow+\infty}\left(K_{n}-\theta(g) K_{n}\right)$. L'inégalité (2) donne 
Vol. 80 (2005) Phénomènes de rang 1 pour les groupes de difféomorphismes du cercle 363

$M=\sup _{h \in \Gamma^{1}}\|c(h)\| \geq \varepsilon \lim \sup _{n}\left\|K_{n}\right\|$, et donc limsup $\sup _{n}\left\|K_{n}\right\| \leq M / \varepsilon$. Par suite, $\|c(g)\| \leq \lim \sup _{n \rightarrow+\infty}\left(\left\|K_{n}\right\|+\left\|\theta(g) K_{n}\right\|\right) \leq 2 M / \varepsilon$ pour tout $g \in \Gamma$. Le cocycle $c$ est donc uniformément borné, et par le lemme du centre de Tits (voir [18]), il est cohomologiquement trivial. Ceci termine la démonstration.

Nous donnons ci-dessus un énoncé du théorème de super-rigidité en cohomologie réduite dû à Y. Shalom. Nous signalons cependant que nous n'utiliserons pas ce théorème dans toute sa puissance. En effet, dans nos applications nous nous ramènerons assez rapidement au cas où il n'y a pas presque des vecteurs invariants pour l'action unitaire correspondante. Or, il se trouve que dans ce cas les cohomologies réduite et continue coïncident, et le théorème de super-rigidité devient bien plus élémentaire dans ce dernier contexte. Néanmoins, nous avons préféré présenter nos résultats en termes de la cohomologie réduite car nous partageons le principe exprimé au §II de [35], suivant lequel «le bon contexte de présentation est celui de la cohomologie réduite (voir le commentaire précédant la proposition 1.22 dans [35]).

Théorème ([31])). Soient $G=G_{1} \times \cdots \times G_{k}$ un groupe topologique compactement engendré et $\Gamma$ un réseau dans $G$ satisfaisant les propriétés du cadre général. Si $\theta: \Gamma \rightarrow U(\mathcal{H})$ est une représentation unitaire et $c$ un cocycle associé, alors $c$ est cohomologue dans $\overline{\mathrm{H}}^{1}(\Gamma, \theta) \grave{a}$ un cocycle de la forme $c_{0}+c_{1}+\cdots+c_{k}$ qui satisfait :

(i) $c_{0}$ est à valeurs dans l'espace $\mathscr{H}_{0}$ des vecteurs $\theta(\Gamma)$-invariants, et il s'étend continûment en un cocycle (par rapport à la représentation unitaire triviale) de $G$ à valeurs dans $\mathscr{H}_{0}$;

(ii) pour tout $i \in\{1, \ldots, k\}$ le cocycle $c_{i}$ est à valeurs dans un sous-espace $\mathscr{H}_{i}$ de $\mathscr{H}$ qui est $\theta(\Gamma)$-invariant, et sur lequel l'action affine $\theta+c_{i}$ s'étend continûment en une action affine de $G$ qui se factorise sur $G_{i}$.

Remarquons que, si $\theta$ n'a pas de vecteur invariant non nul, alors la composante $c_{0}$ ci-dessus est triviale. De plus, d'après le lemme de Delorme, si $\theta$ n'a pas presque des vecteurs invariants, alors $c$ est en fait cohomologue (dans $\left.\mathrm{H}^{1}(\Gamma, \theta)\right)$ au cocycle $c_{1}+\cdots+c_{k}$.

Ce résultat remarquable a été obtenu par Y. Shalom en s'inspirant de la preuve du théorème des sous-groupes distingués de G. Margulis. Nous n'avons malheureusement pas assez de place pour en dire plus. Nous nous contenterons de rappeler l'un des lemmes clés de la fin de sa démonstration, lequel sera utilisé plus loin pour étendre des homomorphismes. Pour cela, rappelons qu'un groupe topologique $\mathrm{H}$ est séquentiellement complet si toute suite $\left(h_{n}\right)$ de $\mathrm{H}$ vérifiant $\lim _{m, n \rightarrow+\infty} h_{m}^{-1} h_{n}=\mathrm{id}_{\mathrm{H}}$ converge dans $\mathrm{H}$. Le lemme ci-dessus s'appuie sur le fait (facile à vérifier) qu' une application uniformément continue définie sur un sous-espace d'un espace métrique séparable et à valeurs dans un groupe topologique Hausdorff séquentiellement complet s'étend continûment à la fermeture de ce sous-espace. 
Lemme 2.5. Soient $G$ et $\Gamma$ deux groupes comme dans le cadre général, et soit $\phi: \Gamma \rightarrow$ $\mathrm{H}$ un homomorphisme, où $\mathrm{H}$ est un groupe topologique Hausdorff séquentiellement complet. Supposons qu'il existe $i \in\{1, \ldots, k\}$ tel que pour toute suite $\left(g_{n}\right)$ dans $\Gamma$ vérifiant $\lim _{n \rightarrow+\infty} \operatorname{pr}_{i}\left(g_{n}\right)=\mathrm{id}_{G_{i}}$, on a $\lim _{n \rightarrow+\infty} \phi\left(g_{n}\right)=\mathrm{id}_{\mathrm{H}}$. Alors $\phi$ s'étend continûment en un homomorphisme de $G$ vers $\mathrm{H}$ qui se factorise sur $G_{i}$.

\section{Le cocycle de Liouville}

3.1. Annulation du cocycle de Liouville en cohomologie continue. Nous nous proposons de donner dans la suite une formulation plus conceptuelle de la méthode introduite dans [27]. Pour cela, rappelons d'abord que la mesure de Liouville Lv sur $S^{1} \times S^{1}$ est celle dont la fonction densité est

$$
(x, y) \mapsto \frac{1}{4 \sin ^{2}\left(\frac{x-y}{2}\right)} .
$$

Cette mesure a une masse totale infinie. De plus, elle est invariante par $\operatorname{PSL}(2, \mathbb{R})$, car pour toute quadruple de points $a<b<c<d<a$ cycliquement ordonnés sur le cercle on a

$$
\operatorname{Lv}([a, b] \times[c, d])=\log ([a, b, c, d]),
$$

où $[\cdot, \cdot, \cdot, \cdot]$ désigne le birapport. La mesure $L v$ induit une mesure de Radon sur l'espace des géodésiques non orientées du disque de Poincaré (un courant géodésique).

Désignons par $\mathscr{H}=\mathscr{L}_{\mathbb{R}}^{2, \Delta}\left(\mathrm{S}^{1} \times \mathrm{S}^{1}, L v\right)$ l'espace des fonctions $K$ de carré intégrable qui satisfont presque-partout l'égalité $K(x, y)=K(y, x)$. Pour le groupe $\operatorname{Diff}_{+}^{1+\alpha}\left(\mathrm{S}^{1}\right), \alpha>1 / 2$, il existe un cocycle naturel associé à la représentation régulière $\theta$ sur $\mathscr{H}$. Ce cocycle de Liouville $c$ correspond au cobord formel associé à la fonction constante égale à 1 . Plus concrétement,

$$
c\left(g^{-1}\right)(x, y)=1-\left|\sin \left(\frac{x-y}{2}\right)\right|\left[g^{\prime}(x) g^{\prime}(y)\right]^{1 / 2} /\left|\sin \left(\frac{g(x)-g(y)}{2}\right)\right| .
$$

Le fait que $c(g)$ appartient à $\mathscr{H}$ dès que $\alpha>1 / 2$ est une remarque dûe essentiellement à G. Segal et A. Reznikov. Ceci reste valable lorsque $g$ est de classe $\mathrm{C}^{1}$ et sa dérivée appartient à l'espace de Sobolev $\mathscr{H}^{1 / 2+\varepsilon}\left(\mathrm{S}^{1}\right)$, avec $\varepsilon>0$ (voir [32]).

Supposons que $\Gamma$ soit un sous-groupe de $\operatorname{Diff}_{+}^{1+\alpha}\left(S^{1}\right), \alpha>1 / 2$, tel que la restriction du cocycle de Liouville à $\Gamma$ soit triviale (en cohomologie continue). Il existe alors une fonction $K \in \mathscr{H}$ telle que $c(g)=K-\theta(g) K$ pour tout $g \in \Gamma$. Ceci se traduit par le fait que la mesure $L v_{K}$ sur $S^{1} \times S^{1}$ donnée par $d L v_{K}=[1-K]^{2} d L v$ est invariante par $\Gamma$. 
Vol. 80 (2005) Phénomènes de rang 1 pour les groupes de difféomorphismes du cercle 365

Fixons une quadruple quelconque de points $a<b<c<d<a$ cycliquement ordonnés sur le cercle. Puisque pour tout $g \in \Gamma$ on a

$$
L v_{K}([a, b] \times[c, d])=\int_{a}^{b} \int_{c}^{d}[1-K(r, s)]^{2} d L v,
$$

d'après l'inégalité triangulaire et l'égalité (3) on obtient

$$
\left|\sqrt{L v_{K}([a, b] \times[c, d])}-\sqrt{\log ([a, b, c, d])}\right| \leq\|K\|_{2} .
$$

Par le même argument,

$$
\left|\sqrt{L v_{K}([g(a), g(b)] \times[g(c), g(d)])}-\sqrt{\log ([g(a), g(b), g(c), g(d)])}\right| \leq\|K\|_{2} .
$$

Puisque $L v_{K}$ est invariante par $g$,

$$
|\sqrt{\log ([g(a), g(b), g(c), g(d)])}-\sqrt{\log ([a, b, c, d])}| \leq 2\|K\|_{2} .
$$

Donc, si $[a, b, c, d]=2$ alors

$$
[g(a), g(b), g(c), g(d)] \leq \exp \left(\left[\sqrt{\log (2)}+2\|K\|_{2}\right]^{2}\right) .
$$

Nous en déduisons le lemme suivant.

Lemme 3.1. Si $\Gamma$ est un sous-groupe de $\operatorname{Diff}_{+}^{1+\alpha}\left(\mathrm{S}^{1}\right), \alpha>1 / 2$, tel que la restriction du cocycle de Liouville à $\Gamma$ est cohomologiquement triviale, alors il existe $M>0$ telle que pour tout $g \in \Gamma$ et toute quadruple de points $a<b<c<d<a$ vérifiant $[a, b, c, d]=2$, on $a[g(a), g(b), g(c), g(d)] \leq 2 M$.

Les sous-groupes de Homéo $\mathrm{O}_{+}\left(\mathrm{S}^{1}\right)$ vérifiant la conclusion du lemme précédent sont appelés uniformément quasi-symétriques (ils sont appelés $M$-uniformément quasisymétriques si l'on veut insister sur la constante $M$ ). Ces groupes furent étudiés dans [20] et [21] par A. Hinkkanen, qui s'intéressait au problème de savoir s'ils sont toujours quasi-symétriquement conjugués à des sous-groupes de $\operatorname{PSL}(2, \mathbb{R})$. L'une des motivations de ce problème était le fait que la version en dimension 2 (remplacer «quasi-symétrique»par «quasi-conforme» et le «cercle»par la «sphère») était déjà connue (voir [36]). A. Hinkkanen n'a pas complètement résolu cette question, mais il y a répondu par l'affirmative dans plusieurs cas. Quelque temps après, et à l'aide du théorème de convergence (voir [8] et [14]), on a démontré que la réponse est affirmative si l'on considère seulement des conjugaisons topologiques. Bien que le problème de la conjugaison quasi-symétrique reste encore ouvert, ce résultat suffit pour établir la proposition suivante, énoncée toutefois sur une forme un peu plus forte dans [27] (voir [30] pour la preuve de la version générale). 
Proposition 3.2. Si $\Gamma$ est un sous-groupe de $\operatorname{Diff}_{+}^{1+\alpha}\left(\mathrm{S}^{1}\right), \alpha>1 / 2$, tel que la restriction du cocycle de Liouville à $\Gamma$ est cohomologiquement triviale, alors $\Gamma$ est topologiquement conjugué à un sous-groupe de $\operatorname{PSL}(2, \mathbb{R})$.

Un point remarquable de la proposition précédente consiste en ce que la classe de différentiabilité démandée est plus petite que $\mathrm{C}^{2}$ : le théorème de Denjoy n'est pas a priori valable! (voir le Chapitre X de [19]). Le fait d'avoir obtenu une vraie conjugaison topologique et pas seulement une semiconjugaison est donc relié à des propriétés de l'action du groupe en général, et pas à celles d'un seul de ses éléments.

3.2. Une application pour les paires avec la propriété (T) relative. Le but de ce paragraphe est de donner la démonstration du théorème A. Pour cela, reprenons la technique du paragraphe précédent. Le cocycle de Liouville considéré induit une représentation affine de $\Gamma \operatorname{sur} \mathcal{L}_{\mathbb{R}}^{2, \Delta}\left(\mathrm{S}^{1} \times \mathrm{S}^{1}, L v\right)$. Si $\left(\Gamma, \Gamma_{0}\right)$ possède la propriété $(\mathrm{T})$ relative alors cette représentation admet un vecteur invariant par $\Gamma_{0}$, et les arguments $\mathrm{du} \$ 3.1$ (voir la proposition 3.2) montrent que le groupe $\Gamma_{0}$ est topologiquement conjugué à un sous-groupe du groupe de Möbius.

La propriété (T) relative est stable par des extensions centrales finies. Ainsi, l'argument de passage au revêtement à trois feuillets de la preuve du lemme 3.3 de [27] montre que $\Gamma_{0}$ est en fait topologiquement conjugué à un sous-groupe du groupe des rotations. Pour que le texte soit autocontenu, nous rappelons cet argument, dû à D. Witte Morris.

Considérons le revêtement à trois feuillets du cercle. Sur ce revêtement $\hat{S}^{1}$ agit (par difféomorphismes de classe $\mathrm{C}^{1+\alpha}$ ) une extension $\hat{\Gamma}$ de $\Gamma$ de la forme

$$
0 \longrightarrow \mathbb{Z} / 3 \mathbb{Z} \longrightarrow \hat{\Gamma} \longrightarrow \Gamma \longrightarrow 0 .
$$

Si l'on désigne par $\hat{\Gamma}_{0}$ la préimage de $\Gamma_{0}$ dans $\hat{\Gamma}$, alors du fait que la paire $\left(\Gamma, \Gamma_{0}\right)$ a la propriété $(\mathrm{T})$ relative et que $\mathbb{Z} / 3 \mathbb{Z}$ est un groupe fini, la paire $\left(\hat{\Gamma}, \hat{\Gamma}_{0}\right)$ vérifie encore la propriété $(\mathrm{T})$ relative (voir la page 9 de [18] pour l'idée de la preuve de cette affirmation). Comme $\hat{\mathrm{S}}^{1}$ s'identifie au cercle, l'argument plus haut montre que le groupe $\hat{\Gamma}_{0}$ lui aussi est topologiquement conjugué à un sous-groupe de $\operatorname{PSL}(2, \mathbb{R})$. Or, si $g$ est un élément de $\Gamma_{0}$ qui fixe un point du cercle initial, alors l'une de ses préimages dans $\hat{\Gamma}_{0}$ fixe trois points de $\hat{\mathrm{S}}^{1}$ par l'action induite (voir la figure 1). Ceci implique évidemment que $\Gamma_{0}$ est en fait topologiquement conjugué à un sous-groupe de $\operatorname{SO}(2, \mathbb{R})$.

Revenons à la preuve du théorème $\mathrm{A}$. Si le groupe $\Gamma_{0}$ n'est pas fini, alors il est topologiquement conjugué à un groupe dense de rotations. Or, il est facile de voir que le normalisateur dans $\mathrm{Homéo}_{+}\left(\mathrm{S}^{1}\right)$ d'un sous-groupe dense de $\mathrm{SO}(2, \mathbb{R})$ coïncide avec le groupe des rotations. Par suite, $\Gamma$ est topologiquement conjugué à un sousgroupe de $\mathrm{SO}(2, \mathbb{R})$, ce qui achève la démonstration. 
Vol. 80 (2005) Phénomènes de rang 1 pour les groupes de difféomorphismes du cercle 367

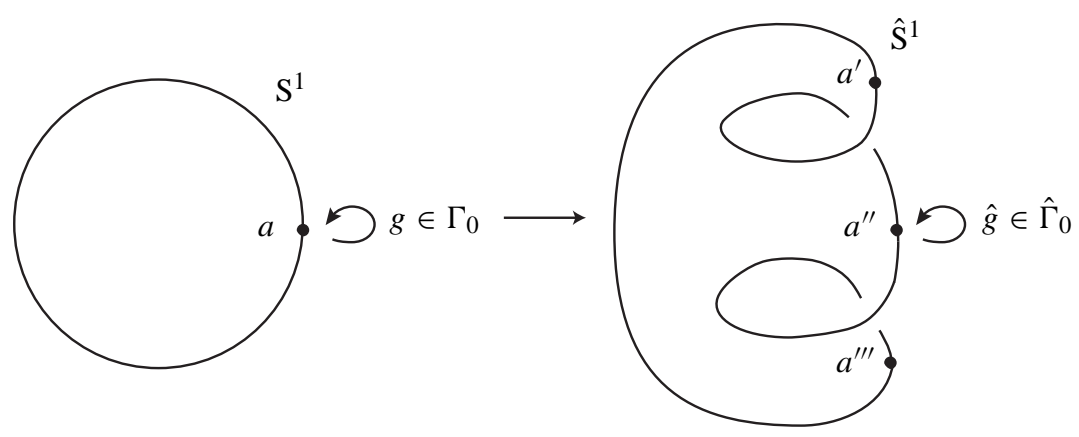

Figure 1

Remarque 3.3. Le théorème $\mathrm{A}$ et ce paragraphe en général ne font que rendre plus naturelle la question suivante : quels sont les sous-groupes de $\operatorname{Diff}_{+}^{1+\alpha}\left(\mathrm{S}^{1}\right), \alpha>1 / 2$, pour lesquels l'action affine associée à la restriction du cocycle de Liouville est géométriquement propre ? Par exemple, ce n'est pas le cas pour le (lissage du) groupe de Thompson G (voir [17]), mais ce groupe est encore (T)-moyennable (voir [12]). La même question se pose pour l'extension finie $\hat{\Gamma}$ de $\Gamma$ qui agit sur le revêtement à trois feuillets du cercle original.

3.3. Annulation en cohomologie réduite. Soient $\alpha>1 / 2$ et $\Gamma$ un sous-groupe de $\operatorname{Diff}_{+}^{1+\alpha}\left(S^{1}\right)$. Nous savons d'après le $\$ 3.1$ que si la restriction du cocycle de Liouville à $\Gamma$ est triviale (en cohomologie continue), alors $\Gamma$ est topologiquement conjugué à un sous-groupe de PSL $(2, \mathbb{R})$. Dans la suite, nous allons étudier le cocycle de Liouville en cohomologie réduite à l'aide du lemme de Delorme.

Lemme 3.4. Supposons que le cocycle de Liouville restreint à $\Gamma$ soit non nul en cohomologie continue mais nul en cohomologie réduite. Alors il existe une mesure de probabilité sur le cercle qui est invariante par $\Gamma$.

Preuve. D'après le lemme de Delorme, si $c$ est nul en cohomologie réduite et non nul en cohomologie continue, alors $\theta$ possède presque des vecteurs invariants. Autrement dit, il existe une suite $\left(K_{n}\right)$ de vecteurs unitaires de $\mathcal{L}_{\mathbb{R}}^{2, \Delta}\left(S^{1} \times S^{1}, L v\right)$ telle que pour tout $g \in \Gamma$, la valeur de $\left\|K_{n}-\theta(g) K_{n}\right\|$ converge vers zéro lorsque $n$ tend vers l'infini. Définisons une suite $\left(\mu_{n}\right)$ de mesures de probabilité sur $S^{1}$ par

$$
\mu_{n}(A)=\int_{\mathrm{S}^{1}} \int_{A} K_{n}^{2}(x, y) d L v
$$


Pour toute fonction continue $\varphi: S^{1} \rightarrow \mathbb{R}$ on a

$$
\begin{aligned}
& \left|\mu_{n}(\varphi)-\phi(g)_{*}\left(\mu_{n}\right)(\varphi)\right| \leq\|\varphi\|_{\mathscr{L}^{\infty}} \int_{\mathrm{S}^{1}} \int_{\mathrm{S}^{1}}\left|K_{n}^{2}-\left(\theta(g) K_{n}\right)^{2}\right| d L v \\
& \leq\|\varphi\|_{\mathscr{L}^{\infty}}\left\|K_{n}+\theta(g) K_{n}\right\|_{\mathscr{L}^{2}}\left\|K_{n}-\theta(g) K_{n}\right\|_{\mathscr{L}^{2}} \\
& \leq 2\|\varphi\|_{\mathscr{L}^{\infty}}\left\|K_{n}-\theta(\mathrm{g}) K_{n}\right\|_{\mathscr{L}^{2}} .
\end{aligned}
$$

On déduit que $\left|\mu_{n}(\varphi)-\phi(g)_{*}\left(\mu_{n}\right)(\varphi)\right|$ tend vers zéro lorsque $n$ tend vers l'infini. Ainsi, si $\mu$ est un point d'adhérence de $\left(\mu_{n}\right)$, alors $\mu$ est une mesure de probabilité sur $\mathrm{S}^{1}$ invariante par $\Gamma$.

Remarque 3.5. Notons que si $\theta$ a un vecteur invariant non nul $K \in \mathcal{L}_{\mathbb{R}}^{2, \Delta}\left(\mathrm{S}^{1} \times\right.$ $\mathrm{S}^{1}, L v$ ), alors quitte à remplacer $K$ par $K /\|K\|$, la mesure $\mu_{K}$ est une mesure de probabilité du cercle qui est invariante par $\Gamma$.

\section{Super-rigidité pour les réseaux de rang supérieur}

4.1. Prolongement de l'action. Plaçons-nous sous les hypothèses du théorème $\mathrm{B}$. Pour chaque fonction $K \in \mathscr{H}=\mathcal{L}_{\mathbb{R}}^{2, \Delta}\left(\mathrm{S}^{1} \times \mathrm{S}^{1}, L v\right)$ de norme 1 , notons $\mu_{K}$ la mesure de probabilité sur $S^{1}$ obtenue en projettant sur la première coordonnée. Plus précisément,

$$
\mu_{K}(A)=\int_{\mathrm{S}^{1}} \int_{A} K^{2}(x, y) d L v .
$$

Désignons par mes l'application mes $(K)=\mu_{K}$ définie sur la sphère unité de $\mathscr{H}$ et à valeurs dans l'espace des mesures de probabilité du cercle absolument continues par rapport à la mesure de Lebesgue. Remarquons que l'on aurait pu définir l'application mes en projettant sur la deuxième coordonnée, mais étant donnée la propriété de symétrie des fonctions de $\mathcal{L}_{\mathbb{R}}^{2, \Delta}\left(\mathrm{S}^{1} \times \mathrm{S}^{1}, L v\right)$, ceci revient au même.

L'application mes est équivariante par rapport à $\Gamma$, dans le sens que pour tout $g \in \Gamma$ et pour toute fonction $K \in \mathcal{L}_{\mathbb{R}}^{2, \Delta}\left(\mathrm{S}^{1} \times \mathrm{S}^{1}, L v\right)$ de norme 1 ,

$$
\operatorname{mes}(\theta(g) K)=\phi(g)_{*}(\operatorname{mes}(K)) .
$$

Supposons que $\phi(\Gamma)$ ne fixe aucune mesure de probabilité sur le cercle et que $\phi(\Gamma)$ ne soit pas conjugué à un sous-groupe de $\operatorname{PSL}(2, \mathbb{R})$. Dans ce cas, d'après le $\S 3$, le théorème de super-rigidité de Shalom fournit une famille $\left\{\mathscr{H}_{1}, \ldots, \mathscr{H}_{k}\right\}$ de sousespaces $\theta(\Gamma)$-invariants de $\mathscr{H}=\mathcal{L}_{\mathbb{R}}^{2, \Delta}\left(\mathrm{S}^{1} \times \mathrm{S}^{1}, L v\right)$, et des cocycles $c_{i}: \Gamma \rightarrow \mathscr{H}_{i}$, dont au moins l'un d'entre eux est non identiquement nul, de sorte que sur chaque $\mathscr{H}_{i}$ la représentation affine associée à $c_{i}$ s'étend continûment à $G$ et se factorise sur $G_{i}$. Fixons un indice $i \in\{1, \ldots, k\}$ tel que $\mathscr{H}_{i}$ soit non trivial. Nous affirmons que l'image de la sphère unité de $\mathscr{H}_{i}$ par l'application mes consiste d'au moins deux 
mesures distinctes. En effet, si cette image était identiquement égale à une mesure mes $(K)$ alors, d'après la propriété d'équivariance (4) et du fait que $\mathscr{H}_{i}$ est un sousespace $\theta(\Gamma)$-invariant, mes $(K)$ serait une mesure de probabilité sur le cercle invariante par $\Gamma$, contredisant notre hypothèse.

Fixons une base Hilbertienne (orthonormée) $\left\{K_{1}, K_{2}, \ldots\right\}$ de $\mathscr{H}_{i}$, et posons

$$
\bar{K}=\sum_{n} \frac{\left|K_{n}\right|}{2^{n}}, \quad K=\frac{\bar{K}}{\|\bar{K}\|} .
$$

La mesure de probabilité $\mu_{K}$ de $\mathrm{S}^{1}$ est une sorte de «mesure à support maximal» parmi les mesures obtenues en projettant des fonctions de $\mathscr{H}_{i}$. Elle est sans atome et absolument continue par rapport à la mesure de Lebesgue. Si l'on désigne par K la fermeture du support de $\mu_{K}$, alors $\mathrm{K}$ est un ensemble compact sans points isolés. De plus, étant donné que $\mathscr{H}_{i}$ est $\theta(\Gamma)$-invariant, l'ensemble $\mathrm{K}$ est invariant par $\Gamma$, et puisque $\phi(\Gamma)$ ne fixe aucune mesure de probabilité du cercle, K n'est pas réduit à une réunion finie disjointe de sous-intervalles fermés de $S^{1}$.

$\mathrm{Si}$ K n'est pas tout le cercle, retirons chaque composante connexe de $S^{1} \backslash \mathrm{K}$, et puis identifions ses extrémités. Par ce procédé on obtient un cercle topologique $S_{K}^{1}$, sur lequel l'action originale de $\Gamma$ induit une action par homéomorphismes directs. Remarquons cependant que les orbites de cette action induite ne sont pas forcément denses : l'ensemble K ne coïncide pas nécessairement avec le minimal exceptionnel de l'action originale (voir [29]). Lorsque K est tout le cercle, notons encore $S_{K}^{1}=S^{1}$. Quelque soit le cas, le cercle $S_{\mathrm{K}}^{1}$ hérite d'une structure métrique naturelle : on peut le paramétrer en utilisant la mesure $\mu_{K}$.

Pour $g \in \Gamma$ notons $\phi_{\mathrm{K}}(g)$ l'homéomorphisme de $S_{\mathrm{K}}^{1}$ induit par $g$. Fixons une fonction $K^{\prime}$ de la sphère unité de $\mathscr{H}_{i}$ telle que la mesure $\mu_{K^{\prime}}$ soit distincte de $\mu_{K}$, et désignons par $\Gamma_{\mu_{K}}$ (resp. $\Gamma_{\mu_{K^{\prime}}}$ ) le groupe des homéomorphismes directs de $\mathrm{S}_{\mathrm{K}}^{1}$ qui préservent la mesure (induite sur $S_{\mathrm{K}}^{1}$ par) $\mu_{K}$ (resp. $\mu_{K^{\prime}}$ ). Remarquons que le groupe $\Gamma_{\mu_{K}}$ est topologiquement conjugué au groupe des rotations. Si $\left(g_{n}\right)$ est une suite d'éléments de $\Gamma$ telle que $\lim _{n \rightarrow+\infty} \operatorname{pr}_{i}\left(g_{n}\right)=\mathrm{id}_{G_{i}}$, alors la valeur de $\left\|\theta\left(g_{n}\right) K-K\right\|$ tend vers zéro lorsque $n$ tend vers l'infini, et de même pour $\left\|\theta\left(g_{n}\right) K^{\prime}-K^{\prime}\right\|$. Un argument analogue à celui de la preuve du lemme 3.4 montre que $\left(\phi_{\mathrm{K}}\left(g_{n}\right)\right)_{*}\left(\mu_{K}\right)$ (resp. $\left.\left(\phi_{\mathrm{K}}\left(g_{n}\right)\right)_{*}\left(\mu_{K^{\prime}}\right)\right)$ tend vers $\mu_{K}$ (resp. vers $\left.\mu_{K^{\prime}}\right)$ lorsque $n$ tend vers l'infini. D'après la première de ces convergences on conclut aisément que la suite $\left(\phi_{\mathrm{K}}\left(g_{n}\right)\right)$ possède des points d'adhèrence dans Homéo $\mathrm{O}_{+}\left(\mathrm{S}_{\mathrm{K}}^{1}\right)$, et d'après ces deux convergences on déduit que tous ces homéomorphismes limites sont contenus dans $\Gamma_{\mu_{K}} \cap \Gamma_{\mu_{K^{\prime}}}$. Puisque $\mu_{K^{\prime}}$ est distincte de $\mu_{K}$ et son support est contenu dans celui de $\mu_{K}$, le groupe $\Gamma_{\mu_{K}} \cap \Gamma_{\mu_{K^{\prime}}}$ est strictement contenu dans $\Gamma_{K}$. Tout sous-groupe non dense du groupe des rotations étant fini et $\Gamma_{\mu_{K}} \cap \Gamma_{\mu_{K^{\prime}}}$ étant un sous-groupe fermé de Homéo $+\left(\mathrm{S}_{\mathrm{K}}^{1}\right)$, le groupe $\Gamma_{\mu_{K}} \cap \Gamma_{\mu_{K^{\prime}}}$ doit nécessairement être fini.

Désignons par Hl'ensemble des $h \in$ Homéo $_{+}\left(\mathrm{S}_{\mathrm{K}}^{1}\right)$ tels que $h=\lim _{n \rightarrow+\infty} \phi_{\mathrm{K}}\left(g_{n}\right)$ 
pour une suite $\left(g_{n}\right)$ dans $\Gamma$ vérifiant $\lim _{n \rightarrow+\infty} \operatorname{pr}_{i}\left(g_{n}\right)=\mathrm{id}_{G_{i}}$. À partir de la définition, il est facile de voir que $\mathrm{H}$ est un sous-groupe fermé de Homéo $\mathrm{S}_{+}\left(\mathrm{S}_{\mathrm{K}}^{1}\right)$. De plus, l'argument plus haut montre que $\mathrm{H}$ est contenu dans $\Gamma_{\mu_{K}} \cap \Gamma_{\mu_{K^{\prime}}}$. C'est donc un groupe fini. Notons $r$ l'ordre de $\mathrm{H}$ et, dans le cas où $r>1$, fixons un générateur $h$ de $\mathrm{H}$. Remarquons que $\rho(h) \neq 0$ (où $\rho$ désigne le nombre de rotation). Fixons une suite $\left(g_{n}\right)$ de $\Gamma$ telle que $\lim _{n \rightarrow+\infty} \operatorname{pr}_{i}\left(g_{n}\right)=\operatorname{id}_{G_{i}}$ et $h=\lim _{n \rightarrow+\infty} \phi_{\mathrm{K}}\left(g_{n}\right)$.

Nous allons démontrer que $\mathrm{H}$ est contenu dans le centralisateur de $\phi_{\mathrm{K}}(\Gamma)$ dans Homéo ${ }_{+}\left(\mathrm{S}_{\mathrm{K}}^{1}\right)$. Pour cela, fixons $g \in \Gamma$. Remarquons que $\operatorname{pr}_{i}\left(g^{-1} g_{n} g\right)$ tend aussi vers $\operatorname{id}_{G_{i}}$ lorsque $n$ tend vers l'infini. Par définition, la suite $\left(\Phi_{\mathrm{K}}\left(g^{-1} g_{n} g\right)\right)$ tend vers un élément de $\mathrm{H}$, c'est-à-dire vers $h^{j}$ pour certain $j \in\{1, \ldots, r\}$. À partir de l'égalité $\rho\left(\phi_{\mathrm{K}}\left(g^{-1} g_{n} g\right)\right)=\rho\left(g^{-1} g_{n} g\right)=\rho\left(g_{n}\right)=\rho\left(\phi_{\mathrm{K}}\left(g_{n}\right)\right), n \in \mathbb{N}$, on conclut aisément que $j=1$. Ceci implique que $\phi_{\mathrm{K}}(g)$ commute avec $h$. Puisque $g \in \Gamma$ était un élément arbitraire, le groupe $\mathrm{H}$ centralise $\phi_{\mathrm{K}}(\Gamma)$.

Désignons par $S_{K}^{1} / \sim$ le cercle topologique obtenu en identifiant les points de $S_{K}^{1}$ qui sont dans la même orbite par $\mathrm{H}$. Le cercle $\mathrm{S}_{\mathrm{K}}^{1}$ est un revêtement fini de degré $r$ du cercle $S_{\mathrm{K}}^{1} / \sim$. De plus, la représentation $\phi_{\mathrm{K}}: \Gamma \rightarrow$ Homéo $_{+}\left(S_{\mathrm{K}}^{1}\right)$ induit de manière naturelle une représentation

$$
\tilde{\phi}: \Gamma \rightarrow \text { Homéo }_{+}\left(\mathrm{S}_{\mathrm{K}}^{1} / \sim\right) .
$$

Notons que si $\left(g_{n}\right)$ est une suite de $\Gamma$ telle que $\operatorname{pr}_{i}\left(g_{n}\right)$ tend vers id $G_{i}$ lorsque $n$ tend vers l'infini, alors $\tilde{\phi}\left(g_{n}\right)$ tend vers l'application identité de $S_{\mathrm{K}}^{1} / \sim$. Nous sommes donc sous les hypothèses du lemme 2.5 , lequel nous permet de conclure que $\tilde{\phi}$ s'étend en une représentation $\Phi: G \rightarrow$ Homéo $_{+}\left(\mathrm{S}_{\mathrm{K}}^{1} / \sim\right)$ qui se factorise sur $G_{i}$. Cette représentation $\Phi$ étend $\phi$ à semiconjugaison topologique et à revêtement fini près, et ceci termine la preuve du théorème $\mathrm{B}$.

Remarque 4.1. Notons que d'après la démonstration du théorème $\mathrm{B}$, dans le cas éventuel d'une semiconjugaison topologique, la partie invariante $\mathrm{K}$ a une mesure de Lebesgue positive.

Rappelons que les sous-groupes finis de Homéo $\left(\mathrm{S}^{1}\right)$ sont topologiquement conjugués à des groupes de rotations, et donc à des sous-groupes de $\operatorname{PSL}(2, \mathbb{R})$ (voir [15]). Ainsi, pour démontrer le corollaire du théorème $\mathrm{B}$, nous montrerons que si $\phi(\Gamma)$ préserve une mesure de probabilité sur le cercle et si $\mathrm{H}^{1}\left(\Gamma_{0}, \mathbb{R}\right)=\{0\}$ pour tout sousgroupe distingué et d'indice fini $\Gamma_{0}$ de $\Gamma$, alors $\phi(\Gamma)$ est fini. En effet, si la mesure invariante n'a pas d'atome alors $\phi(\Gamma)$ est semiconjugué à un groupe de rotations. Sinon, $\phi(\Gamma)$ possède une orbite finie. Étant donné que $\Gamma$ et $\Gamma_{0}$ sont de type fini, ceci implique que $\phi(\Gamma)$ est un groupe fini. Dans le cas d'une orbite finie ceci découle du théorème de stabilité de Thurston, tandis que dans le cas d'une semiconjugaison à un groupe de rotations ceci est à peu près évident. La preuve du corollaire est donc terminée. 
Exemple 4.2. On pourrait être tenté de vouloir utiliser le cocycle de contact à l'origine introduit dans l'exemple 2.1 afin d'obtenir un résultat analogue au théorème $\mathrm{B}$ pour les sous-groupes de $\operatorname{Diff}_{+}^{1+\alpha}([0,1])$ qui ne préservent pas de mesure de Radon sur ]0, 1[. Or, un lemme bien connu et attribué à M. Muller et T. Tsuboi permet de se ramener au cas où les difféomorphismes sont tangents à l'identité à l'origine (voir [26] et [38]), et dans ce cas il a été déjà remarqué dans [41] que la représentation régulière correspondante possède presque des vecteurs invariants. Nous montrerons (toujours dans ce même cas) que le cocycle $c$ donné par (1) est en fait nul en cohomologie réduite.

Fixons un sous-groupe de type fini $\Gamma$ dans Diff ${ }_{+}^{1+\alpha}([0,1])$ tel que $g^{\prime}(0)=1$ pour tout $g \in \Gamma$. Pour chaque $n \in \mathbb{N}$ considérons la fonction

$$
K_{n}(x)=\mathcal{X}_{[1 / n, 1]}(x) .
$$

Il est clair que chaque $K_{n}$ appartient à $\mathcal{L}_{\mathbb{R}}^{2}([0,1], d \mu)$. Nous montrerons que si l'on désigne par $c_{n}$ le cobord associé à cette fonction, alors $c_{n}(g)$ converge vers $c(g)$ pour tout $g \in \Gamma$. En effet, en utilisant les estimées de l'exemple 2.1, on vérifie aisément que

$$
\begin{aligned}
\left\|c(g)-\left(K_{n}-\theta(g) K_{n}\right)\right\|_{\mathscr{H}}^{2} & \leq \frac{C}{n^{2 \alpha}}+\left|\int_{g^{-1}(1 / n)}^{g(1 / n)} \frac{d x}{x}\right| \\
& \leq \frac{C}{n^{2 \alpha}}+\left|\log \left(n g^{-1}(1 / n)\right)\right|+|\log (n g(1 / n))| .
\end{aligned}
$$

D'autre part, il existe nécessairement un point $\left.x_{n} \in\right] 0,1 / n\left[\right.$ tel que $\left(g^{-1}\right)^{\prime}\left(x_{n}\right)=$ $n g^{-1}(1 / n)$. Puisque la suite $\left(x_{n}\right)$ converge vers zéro, $\left(g^{-1}\right)^{\prime}\left(x_{n}\right)$ tend vers 1 . En raisonnant de la même manière avec l'expression $n g(1 / n)$, on déduit que $\| c(g)-$ $\left(K_{n}-\theta(g) K_{n}\right) \|^{2}$ tend vers zéro. Ceci montre que $c(g)$ est la limite des $c_{n}(g)=$ $K_{n}-\theta(g) K_{n}$.

Ce qui précède rend naturelle la question de savoir si l'espace de cohomologie réduite $\overline{\mathrm{H}}^{1}(\Gamma, \theta)$ associé à la représentation régulière $\theta$ ci-dessus est trivial ou non. Signalons par ailleurs que le cocycle de Liouville peut encore être défini pour des groupes de difféomorphismes de l'intervalle (lorsque leur classe de différentiabilité est supérieure à $3 / 2$ ). Cependant, il n'est pas difficile de vérifier que, dans le cas de tangence à l'identité à l'origine, la représentation unitaire correspondante possède presque des vecteurs invariants.

4.2. Actions de groupes localement compacts. Rappelons d'abord le théorème de Montgomery et Zippin (voir [25]) : «si $G$ est un groupe topologique localement compact, alors $G$ est un groupe de Lie si et seulement s'il existe un voisinage de l'identité qui ne contient pas de sous-groupe compact non trivial » (c'est-à-dire s'il n'y a pas de sous-groupe compact «petit $\gg$ ). En utilisant ce résultat profond (et difficile) on 
démontre que tout sous-groupe localement compact de Homéo $+\left(\mathrm{S}^{1}\right)$ est un groupe de Lie (réel). En effet, tout sous-groupe compact de Homéo ${ }_{+}\left(\mathrm{S}^{1}\right)$ étant topologiquement conjugué à un groupe de rotations, Homéo $\mathrm{O}_{+}\left(\mathrm{S}^{1}\right)$ ne contient pas de sous-groupe compact «petit» (voir [15]).

Dans l'introduction nous avons signalé que la classification des actions de groupes de Lie connexes par homéomorphismes directs du cercle est bien connue (voir [13] ou [15]). Plus concrétement, on sait que ces actions transitent par des homomorphismes $\operatorname{sur}(\mathbb{R},+), \operatorname{Aff}_{+}(\mathbb{R}), \operatorname{SO}(2, \mathbb{R}), \widetilde{\operatorname{PSL}}(2, \mathbb{R})$ ou $\operatorname{PSL}_{k}(2, \mathbb{R})$ pour certain $k \geq 1$. Cette classification sera essentielle pour la démonstration suivante.

Preuve du théorème $C$. Nous supposons que $\phi(\Gamma)$ n'est pas fini, ce qui d'après la preuve du corollaire du théorème $\mathrm{B}$ équivaut au fait que $\phi(\Gamma)$ ne préserve aucune mesure de probabilité du cercle.

Considérons d'abord le cas où l'on admet l'hypothèse (i). Le cercle $S_{K}^{1}$ s'identifie alors au cercle original $\mathrm{S}^{1}$. Du fait que le noyau de $\phi$ est fini on conclut qu'il existe des suites $\left(g_{n}\right)$ dans $\Gamma$ telles que $\operatorname{pr}_{i}\left(g_{n}\right)$ converge vers $\operatorname{id}_{G_{i}}$ et les $\phi\left(g_{n}\right)$ sont deux à deux distincts. Ceci implique que le groupe de Lie $\Phi\left(G_{i}\right)$ est non discret. D'après la classification précédente, la composante connexe de l'identité de ce groupe $\Phi\left(G_{i}\right)_{0}$ est soit $\mathrm{SO}(2, \mathbb{R})$, soit un sous-groupe d'un produit de groupes des translations, de groupes affines et de groupes conjugués à $\widetilde{P S L}(2, \mathbb{R})$ agissant sur des intervalles ouverts deux à deux disjoints, soit $\operatorname{PSL}_{k}(2, \mathbb{R})$ pour certain $k \geq 1$. Le premier cas ne peut pas se produire, étant donné que $\phi(\Gamma)$ ne fixe aucune mesure de probabilité du cercle. Le deuxième cas ne peut pas se produire non plus, étant donné que les orbites par $\phi(\Gamma)$ sont denses et que $\Phi\left(G_{i}\right)_{0}$ est distingué dans $\Phi\left(G_{i}\right)$ (car l'ensemble des intervalles fixés par $\Phi\left(G_{i}\right)_{0}$ doit être préservé par $\left.\Gamma\right)$. Le groupe $\Phi\left(G_{i}\right)_{0}$ est donc conjugué à $\operatorname{PSL}_{k}(2, \mathbb{R})$ pour certain $k \geq 1$, et puisque ces derniers groupes coïncident avec leurs normalisateurs dans $\mathrm{Homéo}_{+}\left(\mathrm{S}^{1}\right)$, ceci achève la preuve du théorème sous l'hypothèse (i).

Considérons maintenant le cas de l'hypothèse (ii) suivant laquelle $\phi$ est à valeurs dans Diff ${ }_{+}^{\omega}\left(\mathbf{S}^{1}\right)$. Nous avons déjà remarqué que les orbites par l'action de $\Gamma$ sur $\mathrm{S}_{\mathrm{K}}^{1}$ ne sont pas nécessairement denses. Soit $\mathrm{K}$ l'ensemble fermé non vide invariant et minimal de cette dernière action, et considérons l' action induite $\dot{\Phi}: \Gamma \rightarrow \operatorname{Homéo}\left(\mathrm{S}_{\dot{\mathrm{K}}}^{1}\right)$ sur le cercle topologique $S_{\dot{K}}^{1}$ obtenu en retirant les composantes connexes de $S_{K}^{1} \backslash \dot{K}$ et puis en identifiant ses extrémités. Les orbites par $\dot{\Phi}$ sont denses. Ainsi, pour pouvoir appliquer les arguments de la première partie de la preuve, on doit démontrer que le noyau de $\dot{\Phi}$ est fini. Or, ceci est évident, étant donné que les difféomorphismes de $\phi(\Gamma)$ sont analytiques réels, et ses points fixes sont donc isolés (par conséquence, le noyau de la restriction de $\dot{\Phi}$ à $\Gamma$ coïncide avec celui de $\phi$ ).

Considérons finalement le cas de l'hypothèse (iii) suivant laquelle $\Gamma$ vérifie le théorème des sous-groupes distingués de Margulis. De nouveau, nos devons montrer que le noyau de $\dot{\Phi}$ est fini. Or, si c'est ne pas le cas, alors ce noyau est d'indice fini dans 
Vol. 80 (2005) Phénomènes de rang 1 pour les groupes de difféomorphismes du cercle 373

$\Gamma$. Ceci implique que les orbites par $\Gamma$ des points de $\dot{\mathrm{K}}$ sont finies, ce qui est absurde, car les orbites par $\dot{\Phi}$ sont denses. La preuve du théorème $\mathrm{C}$ est donc terminée.

Remarque 4.3. Signalons que dans [35], Y. Shalom a obtenu - comme une autre application de son théorème de super-rigidité cohomologique - un résultat qui sous une forme faible se lit : «sous les hypothèses du cadre général, si $\phi: \Gamma \rightarrow \operatorname{PSL}(2, \mathbb{R})$ est un homomorphisme avec image non métabélienne, alors $\phi$ s'étend à $G$ et se factorise en un homomorphisme de l'un des $G_{i} \gg$. Comme une conséquence de ce fait, sous les hypothèses du théorème $\mathrm{C}$, lorsque l'image $\phi(\Gamma)$ n'est pas finie, l'homomorphisme $\phi$ s'étend toujours (à semiconjugaison topologique et à revêtement fini près) en un homomorphisme de $G$ qui transite par la projection sur l'un des facteurs $G_{i}$.

Remerciements. L'auteur remercie Y. de Cornulier, D. Gaboriau, É. Ghys, C. Lecuire, N. Monod, M. Pichot, B. Sevennec, Y. Shalom et A. Valette pour d'intéressantes discussions sur le sujet.

\section{Références}

[1] Bader, U., Shalom, Y., Factor and normal subgroup theorems for lattices in products of groups. Prépublication, 2004.

[2] Ballmann, W., Eberlein, P., Fundamental groups of manifolds of nonpositive curvature. J. Differential Geometry 25 (1987), 1-22. Zbl 0701.53070 MR 0873453

[3] Benakli, N., Glasner, Y., Automorphisms groups of trees acting locally with affine permutations. Geom. Dedicata 89 (2002), 1-24. Zbl 1001.20018 MR 1890951

[4] Burger, M., Monod, N., Bounded cohomology of lattices in higher rank Lie groups. J. Eur. Math. Soc. (JEMS) 1 (1999), 199-235. Zbl 0932.22008 MR 1694584

[5] Burger, M., Monod, N., Continuous bounded cohomology and applications to rigidity theory. Geom. Funct. Anal. 12 (2002), 219-280. Zbl 1006.22010 MR 1911660

[6] Burger, M., Mozes, S., Lattices in product of trees. Inst. Hautes Études Sci. Publ. Math. 92 (2000), 151-194. Zbl 1007.22013 MR 1839489

[7] Candel, A., Conlon, L., Foliations I. Grad. Stud. Math. 23, Amer. Math. Soc., Providence, RI, 2000. Zbl 0936.57001 MR 1732868

[8] Casson, A., Jungreis, D., Convergence groups and Seifert fibered 3-manifolds. Invent. Math. 118 (1994), 441-456. Zbl 0840.57005 MR 1296353

[9] Chérix, P., Cowling, M., Jolissaint, P., Julg, P., Valette, A., Groups with the Haagerup property. Gromov's a-(T)-menability. Progr. Math. 197, Birkhäuser, Basel 2001. Zbl 1030.43002 MR 1852148

[10] de Cornulier, Y., Relative Kazhdan property. Prépublication, 2005.

[11] Farb, B., Shalen, P., Real-analytic actions of lattices. Invent. Math. 135 (1998), 273-296. Zbl 0954.22007 MR 1666834 
[12] Farley, D., A proper isometric action of Thompson group V on Hilbert space. Internat. Math. Res. Notices 45 (2003), 2409-2414. Zbl 02010461 MR 2006480

[13] Furman, A., Mostow-Margulis rigidity with locally compact targets. Geom. Funct. Anal. 11 (2001), 30-59. Zbl 0984.22008 MR 1829641

[14] Gabai, D., Convergence groups are Fuchsian groups. Ann. of Math. 136 (1992), 447-510. Zbl 0785.57004 MR 1189862

[15] Ghys, É., Groups acting on the circle. Enseign. Math. (2) 47 (2001), 329-407. Zbl 1044.37033 MR 1876932

[16] Ghys, É., Actions de réseaux sur le cercle. Invent. Math. 137 (1999), 199-231. Zbl 0995.57006 MR 1703323

[17] Ghys, É., Sergiescu, V., Sur un groupe remarquable de difféomorphismes du cercle. Comment. Math. Helv. 62 (1987), 185-239. Zbl 0647.58009 MR 0896095

[18] de la Harpe, P., Valette, A., La propriété (T) de Kazhdan pour les groupes localement compacts. Astérisque 175 (1989). Zbl 0759.22001 MR 1023471

[19] Herman, M., Sur la conjugaison différentiable des difféomorphismes du cercle à des rotations. Inst. Hautes Études Sci. Publ. Math. 49 (1979), 5-233.Zbl 0448.58019 MR 0538680

[20] Hinkkanen, A., The structure of some quasisymmetric groups. Mem. Amer. Math. Soc. 83 (1990), 87 pp. Zbl 0707.30018 MR 0948926

[21] Hinkkanen, A., Uniformly quasisymmetric groups. Proc. London Math. Soc. 51 (1985), 318-338. Zbl 0574.30022 MR 0794115

[22] Korevaar, N., Schoen, R., Global existence theorems for harmonic maps to non-locally compact spaces. Comm. Anal. Geom. 5 (1997), 333-387. Zbl 0908.58007 MR 1483983

[23] Monod, N., Continuous bounded cohomology of locally compact groups. Lecture Notes in Math. 1758, Springer-Verlag, Berlin 2001. Zbl 0967.22006 MR 1840942

[24] Monod, N., Shalom, Y., Cocycle superrigidity and bounded cohomology for negatively curved spaces. J. Differential Geometry 67 (2004), 1-61.

[25] Montgomery, D., Zippin, L., Topological Transformation Groups. Interscience Publishers, New York 1955. Zbl 0068.01904 MR 0073104

[26] Muller, M., Sur l'approximation et l'instabilité des feuilletages. Texte non publié, 1982.

[27] Navas, A., Actions de groupes de Kazhdan sur le cercle. Ann. Sci. École Norm. Sup. 35 (2002), 749-758. Zbl 1028.58010 MR 1951442

[28] Navas, A., Groupes de Neretin et propriété (T) de Kazhdan. C. R. Acad. Sci. Paris Sér. I Math. 335 (2002), 789-792. Zbl 1018.22002 MR 1947700

[29] Navas, A., Sur les groupes de difféomorphismes du cercle engendrés par des éléments proches des rotations. Enseign. Math. 50 (2004), 29-68. MR 2084334

[30] Navas, A., Reduction of cocycles and groups of diffeomorphisms of the circle. À paraitre dans Bull. Belgian Math. Soc.

[31] Plante, J., Foliations with measure preserving holonomy. Ann. of Math. 102 (1975), 327-361. Zbl 0314.57018 MR 0391125

[32] Pressley, A., Segal, G., Loop groups. Oxford Mathematical Monographs, The Clarendon Press, Oxford University Press, New York 1986. Zbl 0618.22011 MR 0900587 
Vol. 80 (2005) Phénomènes de rang 1 pour les groupes de difféomorphismes du cercle 375

[33] Rémy, B., Construction de réseaux en théorie de Kac-Moody. C. R. Acad. Sci. Paris Sér. I Math. 6 (1999), 475-478. Zbl 0933.22029 MR 1715140

[34] Reznikov, A., Analytic topology of groups, actions, strings and varietes. Chapter 2 : A theory of groups acting in the circle. Preprint, August 11 (1999), 59-65. Disponible électroniquement : http ://xxx.lpthe.jussieu.fr/find/math/1/au :+Reznikov_A/0/1/0/all/0/1.

[35] Shalom, Y., Rigidity of commensurators and irreducible lattices. Invent. Math. 141 (2000), 1-54. Zbl 0978.22010 MR 1767270

[36] Sullivan, D., On the ergodic theory at infinity of an arbitrary discrete group of hyperbolic motions. In Riemann surfaces and related topics: Proceedings of the 1978 Stony Brook Conference. Ann. of Math. Stud. 97, Princeton University Press, Princeton, N.J., 1981, 465-496. Zbl 0567.58015 MR 0624833

[37] Thurston, W., A generalization of Reeb stability theorem. Topology 13 (1974), 347-352. Zbl 0305.57025 MR 0356087

[38] Tsuboi, T., $\Gamma_{1}$-structures avec une seule feuille. Astérisque 116 (1984), 222-234. Zbl 0551.57014 MR 0755173

[39] Witte, D., Products of similar matrices. Proc. Amer. Math. Soc. 126 (1998), 1005-1015. Zbl 0893.20033 MR 1451837

[40] Witte, D., Arithmetic groups of higher $\mathbb{Q}$-rank cannot act on 1-manifolds. Proc. Amer. Math. Soc. 122 (1994), 333-340. Zbl 0818.22006 MR 1198459

[41] Witte, D., Zimmer, R., Actions of semisimple Lie groups on circle bundles. Geom. Dedicata 87 (2001), 91-121. Zbl 1017.22004 MR 1866844

Received November 24, 2003

Andrés Navas, IHÉS, 35 route de Chartres, 91440 Bures sur Yvette, France, et Universidad de Chile, Las Palmeras 3425, Ñuñoa, Santiago, Chile

E-mail: anavas@ihes.fr, andnavas@uchile.cl 